Int. J. Speleol. 15 (1986), pp. 53-56

\title{
KURZBERICHT DER ERSTEN DEUTSCHEN SPELÄOLOGISCHEN HIMALAYA-EXPEDITION.
}

\section{(PRELIMINARY REPORT OF THE FIRST GERMAN SPELEOLOGICAL EXPEDITION TO THE HIMALAYA)}

\author{
Herbert Daniel Gebauer *
}

\begin{abstract}
The first german speleological expedition to the Himalaya went to the terracesediments of Pokhara, Nepal. New discoveries could be made in the longest cave of the Himalaya (Patalae Chhango or Harpan-River-Cave) and other caves were surveyed for the first time.

Due to the observations is the classification in the lump of conglomerate-caves as pseudokarst-appearences to be questioned.
\end{abstract}

\section{ZUSAMMENFASSUNG}

Die erste deutsche speläologische Himalaya-Expedition führte zu den Terassensedimenten von Pokhara, Nepal. In der bisher größten Höle des Himalaya (Patalae Chhango oder Harpan-River-Cave) konnte Neuland entdeckt werden und andere Objekte wurden zum erstenmal vermessen.

Aufgrund der Beobobachtungen muß die pauschale Einstufung von Konglomerathöhlen als Pseudokarsterscheinungen in Frage gestellt werden.

\section{DAS HÖHLENBILDENDE KONGLOMERAT}

Die Höhlen von Pokhara befinden sich in den Terassensedimenten zwischen den zentralen Himalaya und dem Mahabharat-Lekh. Die Terassen bestehen aus zwei unterschiedlichen Klastischen Sedimenten kalzitgebundener Flußgerölle pleistozäner Herkunft.

* Marktplatz 32, D-7070 Schwäbisch Gmünd 
Die obenliegende Konglomeratbank ist etwa 15 bis $20 \mathrm{~m}$ stark und differenziert modal. Sie besteht aus einem ungeschichteten Durcheinander zwischen automobilgroßen Findlingen bis hin zu staubfeinem Sand aus Phylliten, Granat, Gneisen, Basalt, Kalk und vielem anderen mehr. Die Matrix besteht aus etwa gleichen Teilen Kalzitkristallen und Quarzsand.

Darunter liegt eine mindestens $40 \mathrm{~m}$ starke Konglomeratbank aus feinem Sand mit wenigen gröberen Einlagerungen. Sie ist häufig geschichtet in unterschiedlich widerstandsfähige Schichten, die im allgemeinen nur wenig lithifiziert, spröde und porös sind. Sie besteht aus 50 bis $80 \%$ Kalzitkristallen und-sanden, der Rest sind gerundete Quarzsandkörner, ein wenig Gips und organische Rückstände. Manchmal sind auch tonige Schichten eingelagert, die extrem labil sind.

\section{CHARAKTERISTIK DER KONGLOMERATHÖHLEN}

Da die Höhlen in zwei unterschiedlichen Konglomeraten verlaufen wird das allgemeine Erscheinungsbild der Höhlen dem höhlenbildenden Gestein entsprechend unterschiedlich geprägt.

Klüfte streichen selten eindeutig in gerader Richtung, sondern schlängeln sich eher durch eine nach unten und oben beschränkte Anzahl von Schichten. Auch verlaufen sie gern entlang den Talabbruchkanten und fallen meist senkrecht.

Die widerstandsfähigere obere Konglomeratbank bildet in den Hölen entweder waagrecht hängende Decken, die sich freitragend über viele Meter hinweg von Wand zu Wand ziehen oder statisch ideale Gewölbe, welche abgespannte Schuppen inkadieren oder sich Kiesel für Kiesel sphärisch weiten.

In der unteren Konglomeratbank sind die Decken allenfalls auf kurzer Spannweite waagrecht und meistens als "falsche Gewölbe" aus einander überkragenden Schichtplatten gebildet.

Es finden sich für Konglomerhöhlen unerwartet große Säle und Versturzhallen (chemioklastische Versturzsäle) die manchmal bis knapp unter den Zenith der Kuppeln mit charakteristischen Versturzbergen erfüllt sind, deren Flanken sich konvex den Versturzhallenwänden annähern.

In der oberen Konglomeratbank sind Sinterbildungen, dem 


\section{PATALAE CHHANGO \\ DEVIS FALL}

Tashilling. Pokhara,

Nepal.Asien

SEEHOHE: CO $900 \mathrm{~m} \mathrm{UMNN}$

GESAMTLANGE : $2056.7 \mathrm{~m}$

WIV. DIFFERENZ: $-48.1 \mathrm{~m}$

VERMESSUNG: 6.2. -15.2 .7980$

ECRA-Grod: SB

Vermessung. Entwurt, Ber echnung

und Zeichriuns: D. Gebouer
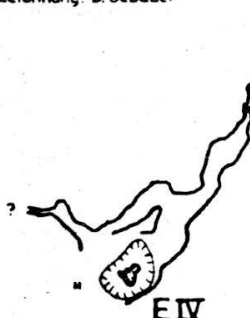

N

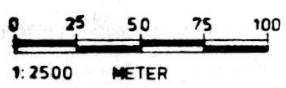

E $\mathbf{Y} / \mathbf{Z}$

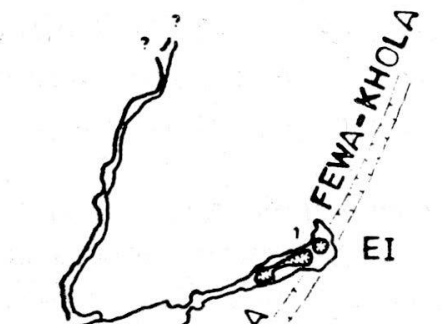

EI

SEE AM 16.2.990

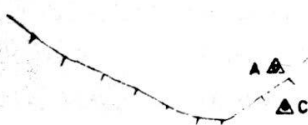

1 Wasserfall. Potalae Chnango

2 Conon unter dem Wisserfall

3 istlicher Nord-Sudgang

westlicher Nord-Südgang

Klookentalle

Gang zum Phusre-Khoio

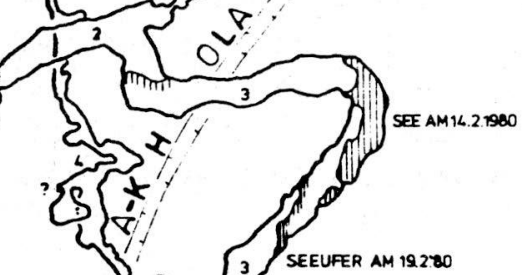

0

(1)

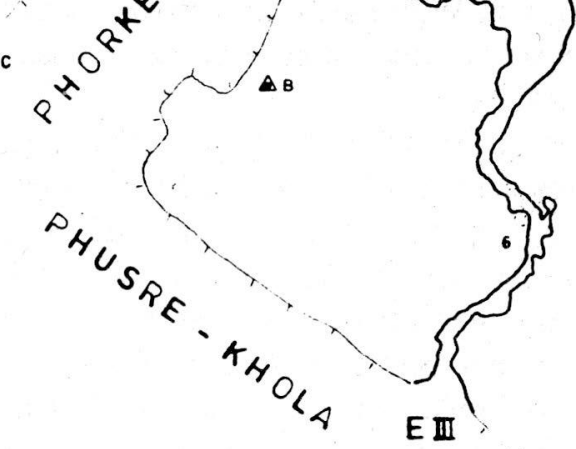


außerordentlich geringen Kalzitanteil entsprechend, selten jedoch in der unteren unerwartet häufig. In erster Linie treten Stalaktiten auf, die in Reihen das Kluftnetz nachzeichnen oder in zusammenhängenden Flächen wachsen, wenn das sinterbildende Wasser flächig aus dem porösen Konglomerat dringt.

Bodenzapfen kommen selten vor und erreichen aber trotz des geringen Kalzitangebots bemerkenswerte Größen. Auch deuten tonnenschwere, sich auflösende Sintermassen auf eine sinterbildendere Zeit hin.

\section{GEDANKEN ZUR HÖHLENBILDUNG IM KONGLOMERATKARST}

Auch die neuere speläologische Literatur hat sich in Bezug auf Konglomerathölen noch nicht aus der aristotelischen Einfalt gelöst, wenn sie ungeachtet der petrographischen Unterschiede zwischen verschiedenen Konglomeraten alle Konglomerathölen pauschal als Pseudokarsterscheinungen abtut und nicht selten so einfältig wie Kyrle schreibt, der Konglomerate zur Bildung großräumiger Höhlen nicht für möglich hält, "weil derartige Höhlenräume, die ein gewisses Raummaß überschreiten, wieder in sich zusammenbrechen" (Theoretische Speläologie, S. 19).

Das von Kyrle gemeinte Raummaß ist in den Hallen des Patalae Chhango gewiß überschritten. Das verzweigte Höhlensystem bezeugt eine echte Karstentwässerung des Konglomerats und allein die Sinterbildungen beweisen die Echtheit des nepalesischen Konglomeratkarsts.

Dennoch verkarsten die meisten Konglomerate nicht im eigentlichen Sinn. Es muß deshalb eine in die Konglomerate hineingeschobene Definitionsgrenze gefunden werden.

Den Ausschlag für die Verkarstungsfähigkeit eines Konglomerats dürfte von den Verkarstungsfähigkeiten des Sediments und des Bindemittels abhängig sein, vom Verhältnis der verkarstungsfähigen Anteile zu den nichverkarstungsfähigen und der räumlichen Verteilung und Beziehung der Konglomeratbestandteile. Auch der dynamische Aspekt muß miteinbezogen werden, da ein losgelöster Kieselstein einen beträchtlich umfangreicheren Weg zum Abtransport benötigt als 'ein aufgelöster Kalkstein. 